\title{
Correction to: V Brazilian consensus guidelines for detection of anti-cell autoantibodies on hep-2 cells
}

Wilson de Melo Cruvinel ${ }^{1 *}$, Luis Eduardo Coelho Andrade 2 , Carlos Alberto von Mühlen ${ }^{3}$, Alessandra Dellavance ${ }^{4}$, Antônio Carlos Ximenes ${ }^{5}$, Carlos David Bichara ${ }^{6}$, Cleonice Bueno ${ }^{7}$, Cristóvão Luis Pitangueira Mangueira ${ }^{8}$, Eloísa Bonfá ${ }^{9}$, Fabiano de Almeida Brito ${ }^{10}$, Fernanda Bull Flumian ${ }^{11}$, Glaucielen Gomes da Silva ${ }^{12}$, Jozelia Rêgo ${ }^{13}$, Lisiane Maria Ericoni dos Anjos ${ }^{14}$, Natasha Slhessarenko ${ }^{15}$, Sandra Gofinet Pasoto ${ }^{16}$, Suzane Pretti Figueiredo Neves ${ }^{17}$, Valéria Valim ${ }^{18}$, Wilton Silva dos Santos ${ }^{19}$ and Paulo Luiz Carvalho Francescantonio ${ }^{1^{*}}$

\section{Correction to: Adv Rheumatol https://doi.org/10.1186/s42358-019-0069-5}

After publication of the original article [1], we were notified that there is a mistake in Fig. 2.

Two codes BAC-1 and BAC-2 are switched in the first and second rows. In the first line the correct one is BAC-2 instead of BAC-1 and in the second line, BAC-1, instead of BAC-2.

The correct version of Fig. 2 can be found below.

\begin{abstract}
Author details
'Pontifícia Universidade Católica de Goiás (PUC Goiás), Escola de Ciências Médicas, Farmacêuticas e Biomédicas, Avenida Universitária 1.440, Setor Universitário, Goiânia, GO CEP 74605-010, Brazil. 'Disciplina de Reumatologia, Escola Paulista de Medicina, Universidade Federal de São Paulo (UNIFESP), Divisão de Imunologia, Fleury Medicina e Saúde, São Paulo, SP, Brazil. ${ }^{3}$ Sociedade Brasileira de Autoimunidade, Porto Alegre, RS, Brazil. ${ }^{4}$ Divisão de Pesquisa, Inovação e Desenvolvimento, Fleury Medicina e Saúde, São Paulo, SP, Brazil. ${ }^{5}$ Diretor Clinico do Hospital Geral de Goiania Alberto Rassi, Goiânia, GO, Brazil. ${ }^{6}$ Faculdade de Medicina Famaz, Amaral Costa Medicina Diagnóstica, Belém, PA, Brazil. 'Laboratórios de Investigação Médica, Hospital das Clínicas, Faculdade de Medicina, Universidade de São Paulo (FM-USP), São Paulo, SP, Brazil. ${ }^{8}$ Departamento de Patologia Clínica e Anatomia Patológica, Hospital Israelita Albert Einstein, São Paulo, SP, Brazil. ${ }^{9} \mathrm{Hospital}$ das Clinicas HCFMUSP, Faculdade de Medicina, Universidade de Sao Paulo, Sao Paulo, SP, Brazil. ${ }^{10}$ Instituto Hermes Pardini, Belo Horizonte, MG, Brazil. ${ }^{11}$ Grupo DASA, São Paulo, SP, Brazil. ${ }^{12}$ Faculdade Carajás, Marabá, PA, Brazil. ${ }^{13}$ Faculdade de Medicina, Universidade Federal de Goiás (UFG), Goiânia, GO, Brazil. ${ }^{14}$ Universidade do Sul de Santa Catarina (UNISUL) e Universidade do
\end{abstract}

Vale do Itajaí (UNIVALI), Florianópolis, SC, Brazil. ${ }^{15}$ Universidade Federal de Mato Grosso (UFMT) e Grupo DASA Cuiabá, Cuiabá, MT, Brazil. ${ }^{16}$ Serviço de Reumatologia e Laboratório de Autoimunidade da Divisão de Laboratório Central do Hospital das Clínicas da Faculdade de Medicina da Universidade de São Paulo (USP), São Paulo, SP, Brazil. ${ }^{17}$ Departamento de Propedêutica Complementar da Faculdade de Medicina, Universidade Federal de Minas Gerais (UFMG), Belo Horizonte, MG, Brazil. ${ }^{18}$ Universidade Federal do Espírito Santo (UFES), Vitória, ES, Brazil. ${ }^{19}$ Escola Superior de Ciências da Saúde do Distrito Federal e Laboratório Sabin, Brasília, DF, Brazil.

Published online: 06 January 2020

\section{Reference}

1. Cruvinel, et al. V Brazilian consensus quidelines for detection of anti-cell autoantibodies on hep-2 cells. Adv Rheumatol. 2019;59:28. https://doi.org/ 10.1186/s42358-019-0069-5.

The original article can be found online at https://doi.org/10.1186/s42358019-0069-5

*Correspondence: melocruvinel@gmail.com; paulo_luiz1@hotmail.com ${ }^{1}$ Pontifícia Universidade Católica de Goiás (PUC Goiás), Escola de Ciências Médicas, Farmacêuticas e Biomédicas, Avenida Universitária 1.440, Setor Universitário, Goiânia, GO CEP 74605-010, Brazil

Full list of author information is available at the end of the article

(c) The Author(s). 2019 Open Access This article is distributed under the terms of the Creative Commons Attribution 4.0 International License (http://creativecommons.org/licenses/by/4.0/), which permits unrestricted use, distribution, and reproduction in any medium, provided you give appropriate credit to the original author(s) and the source, provide a link to the Creative Commons license, and indicate if changes were made. The Creative Commons Public Domain Dedication waiver (http://creativecommons.org/publicdomain/zero/1.0/) applies to the data made available in this article, unless otherwise stated. 


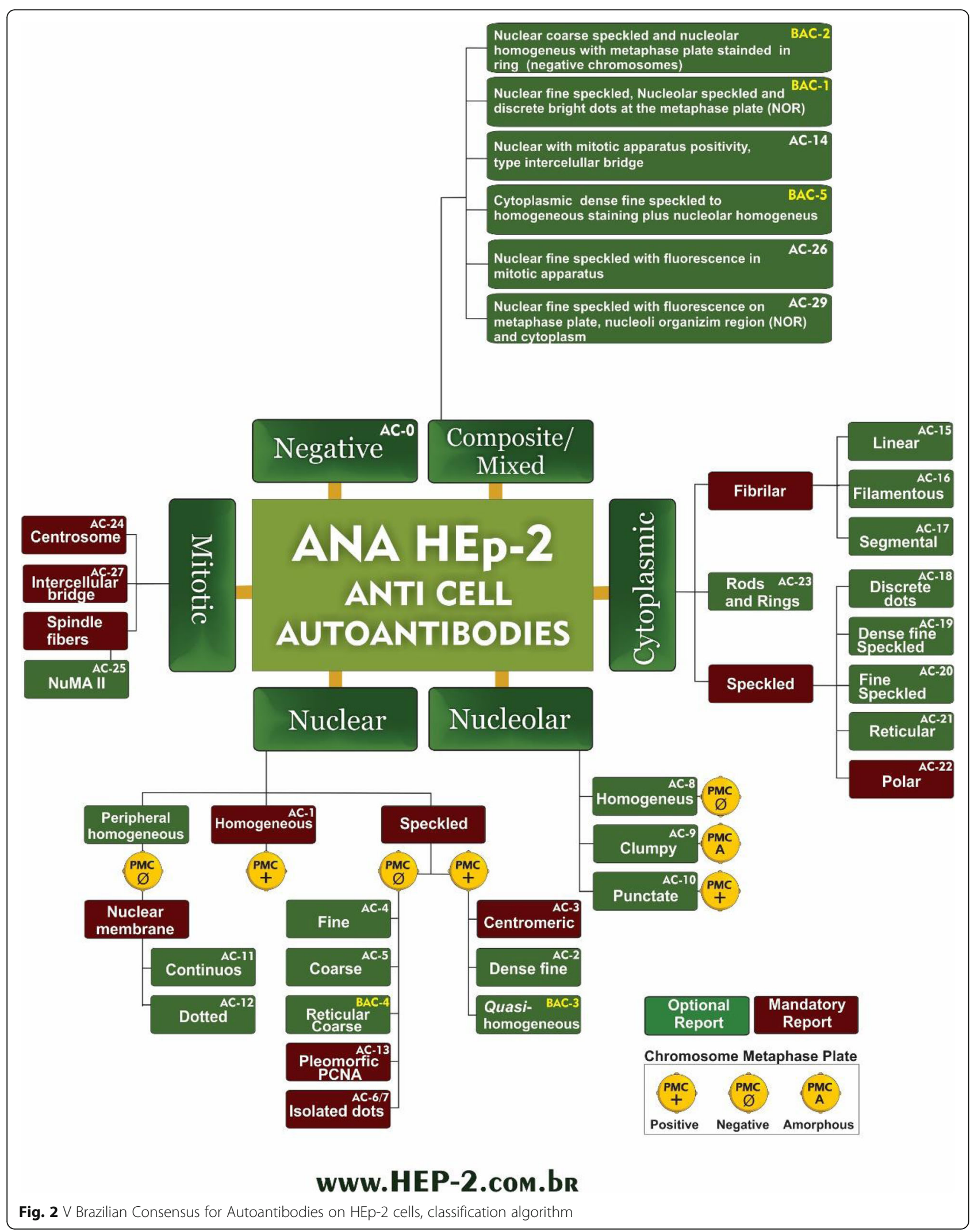

